\title{
NONSMOOTH NEWTON METHODS FOR SET-VALUED SADDLE POINT PROBLEMS
}

\author{
CARSTEN GRÄSER AND RALF KORNHUBER
}

\begin{abstract}
We present a new class of iterative schemes for large scale setvalued saddle point problems as arising, e.g., from optimization problems in the presence of linear and inequality constraints. Our algorithms can be either regarded as nonsmooth Newton-type methods for the nonlinear Schur complement or as Uzawa-type iterations with active set preconditioners. $\mathrm{Nu}-$ merical experiments with a control constrained optimal control problem and a discretized Cahn-Hilliard equation with obstacle potential illustrate the reliability and efficiency of the new approach.
\end{abstract}

\section{INTRODUCTION}

We consider the iterative solution of large scale saddle point problems of the form

$$
u^{*} \in \mathbb{R}^{n}, w^{*} \in \mathbb{R}^{m}: \quad\left(\begin{array}{cc}
F & B^{T} \\
B & -C
\end{array}\right)\left(\begin{array}{l}
u^{*} \\
w^{*}
\end{array}\right) \ni\left(\begin{array}{l}
f \\
g
\end{array}\right),
$$

where $B, C$ are suitable matrices, and the set-valued operator $F=\partial \varphi$ stands for the subdifferential of a strictly convex functional $\varphi$. Such kind of problems typically arise from the discretization of optimization or optimal control problems governed by partial differential equations with inequality constraints (cf., e.g., [34, 47]). In case of a quadratic objective functional, we get

$$
F=A+\partial I_{K}
$$

where $I_{K}$ is denoting the indicator functional of the admissible set $K, A$ is a selfadjoint positive definite, sometimes even diagonal matrix, and $C=0$. Another rich and still growing class of problems of the form (1.1) consists of discretized phase field models, such as Cahn-Hilliard equations [5, 6, 8, 18, 19], PenroseFife equations [10] or Stefan-type problems [50]. For example, discretization of Cahn-Hilliard equations with logarithmic potential leads to the single-valued but singularly perturbed nonlinearity $F(u)=A u+T \log ((1+u) /(1-u))$ where the logarithmic term is understood componentwise. Nonlinearities of the form (1.2) occur as singular limit for vanishing temperature $T$. The matrices $A, C$ are essentially stiffness matrices of the Laplacian with $A$ augmented by a non-local term reflecting mass conservation. Other possible applications include discretized plasticity problems $[21,45]$.

Key words and phrases. set-valued saddle point problems, nonsmooth Newton methods, Uzawa algorithms, active-set preconditioners.

The authors would like to thank the unknown referees for their most valuable comments and suggestions. This work has been funded in part by the Deutsche Forschungsgemeinschaft (DFG) under contract Ko 1806/3-1 and by the DFG Research Center Matheon. 
Saddle point problems of the form (1.1) with single-valued, Lipschitz-continuous nonlinearities $F$ have been considered in [12, 28]. Interior point methods (cf., e.g., $[52,53])$ are based on suitable regularizations of set-valued nonlinearities (1.2). It is not immediately clear, how this strategy should be generalized to singlevalued but singularly perturbed nonlinearities. Existing primal-dual active set methods $[27,48]$ are based on the elimination of the state variables $u_{s}$ and an active set approach to the resulting constrained minimization problem for the controls $u_{c}$. These methods are applicable to (1.1) with $u=\left(u_{s}, u_{c}\right)$, provided that the corresponding partitioning of $B=\left(B_{s}, B_{c}\right)$ generates an invertible matrix $B_{s}$, that the set-valued nonlinearity (1.2) only constrains $u_{c}$, and finally $C=0$. For example, discretized Cahn-Hilliard equations have none of these properties.

The novel approach presented in this paper relies on convexity rather than smoothness. It is motivated by the fact that a variety of practically relevant nonlinearities $F$ can be either inverted in closed form or can be efficiently inverted by multigrid methods. This includes, e.g., the nonlinearities mentioned above $[3,4,26,30,32,33]$.

The basic idea is to reformulate (1.1) as an unconstrained convex minimization problem for the dual unknown $w$. The gradient of the objective functional $h$ is just the nonlinear Schur complement $H$ of $(1.1)$ and thus involves $F^{-1}$. Minimization of $h$ is carried out by well-known gradient-related descent methods (cf., e.g., [38, 39, 40]). Global convergence is enforced by standard Armijo damping [2], for simplicity. We particularly concentrate on nonsmooth Newton or Newton-like methods for nonlinearities of the form (1.2) taking into account that the nonlinear Schur complement $H$ is Lipschitz but not differentiable in the classical sense. We prove global convergence and local exactness. Inexact versions are shown to be globally convergent.

In the special case of discretized optimal control problems with control constraints and diagonal matrix $A$ our algorithms reduce to well-known primal-dual active set methods [23]. Hence, the algorithms presented in this paper can be regarded as a new variational approach to primal-dual active set strategies, thus providing a natural globalization and generalization of these methods. Extensions to single-valued, but singularly perturbed nonlinearities $F$ will be presented in a forthcoming paper [25]. Our approach also sheds new light on well-established algorithms in computational plasticity [51].

From a computational point of view, our algorithms can be reinterpreted as nonlinear Uzawa iterations with active-set preconditioners [24]. For nonlinearities of the form (1.2), each iteration step requires the detection of the actual active set of $u^{\nu}=F^{-1}\left(f-B^{T} w^{\nu}\right)$ (not of $u^{\nu}$ itself!), and the sufficiently accurate evaluation of a corresponding linear saddle point problem (the actual preconditioner). We found in our numerical experiments with a discretized Cahn-Hilliard equation that, for bad initial iterates, the overall computational work was dominated by Armijo damping, because each Armijo test involves the exact evaluation of $F^{-1}$, i.e., the solution of a discrete elliptic obstacle problem. For reasonable initial iterates as obtained, e.g., from the preceding time step, almost no damping was necessary. In this case the (inexact) evaluation of the linear saddle point problem clearly dominated the overall computational cost.

The paper is organized as follows. After some notation and a precise formulation of the assumptions, we derive the equivalent unconstrained minimization problem 
which is fundamental for the rest of this paper. In Section 3, we recall some general convergence results for gradient-related descent methods for unconstrained minimization, including damping strategies and inexact variants. Then, we concentrate on the selection of suitable descent directions for the special case of nonlinearities of the form (1.2). More precisely, we investigate the B-subdifferential of $F$ and later of $H$, giving rise to various nonsmooth Newton-type methods. The main convergence results are collected in Theorems $4.1-4.3$. Section 5 provides a more tangible reformulation of these abstract schemes in terms of quadratic obstacle problems and linear saddle point problems. Inexact evaluation of both of these subproblems and a heuristic damping strategy is also discussed. In our numerical computations, we consider a control constrained optimal control problem and a discretized Cahn-Hilliard equation. We found superlinear convergence and finite termination, supporting our theoretical findings.

\section{Set-Valued SAdDle Point PROBlems}

2.1. General assumptions and notation. Let $\langle\cdot, \cdot\rangle$ denote the euclidian inner product on $\mathbb{R}^{m}$. We equip $\mathbb{R}^{m}$ with the norm $\|\cdot\|_{M}$,

$$
\|x\|_{M}^{2}=\langle M x, x\rangle, \quad x \in \mathbb{R}^{m},
$$

induced by a fixed symmetric, positive definite (s.p.d.) matrix $M \in \mathbb{R}^{m, m}$. Linear mappings will be identified with their matrix representations with respect to the canonical basis vectors $e_{i}$ with the coefficients $\left(e_{i}\right)_{j}=\delta_{i, j}$ (Kronecker- $\delta$ ). Elements $x^{\prime}$ of the dual space $\left(\mathbb{R}^{m}\right)^{\prime}$ will be represented as $x^{\prime}=\langle x, \cdot\rangle$ with suitable $x \in \mathbb{R}^{m}$. Hence, using

$$
\left|x^{\prime}(y)\right|=|\langle x, y\rangle| \leq\left\|M^{-\frac{1}{2}} x\right\|\left\|M^{\frac{1}{2}} y\right\|=\|x\|_{M^{-1}}\|y\|_{M},
$$

the dual space $\left(\mathbb{R}^{m},\|\cdot\|_{M}\right)^{\prime}$ is identified with $\left(\mathbb{R}^{m},\|\cdot\|_{M^{-1}}\right)$.

We impose the following conditions on the saddle point problem (1.1).

(A1) $F=\partial \varphi$ is the subdifferential of a proper, lower semi-continuous, strictly convex functional $\varphi: \mathbb{R}^{n} \rightarrow \overline{\mathbb{R}}=\mathbb{R} \cup\{\infty\}$. The inverse $F^{-1}: \mathbb{R}^{n} \rightarrow \mathbb{R}^{n}$ is single-valued and Lipschitz continuous.

(A2) $C \in \mathbb{R}^{m, m}$ is symmetric, positive semi-definite.

(A3) $B \in \mathbb{R}^{m, n}$.

(A4) The saddle point problem (1.1) has a unique solution.

Nonlinearities $F$ satisfying condition (A1) occur, e.g., in discretized Cahn-Hilliard equations with logarithmic potential [5]. Later on, we will concentrate on the special case

$$
F=A+\partial I_{K}
$$

where $A \in \mathbb{R}^{n, n}$ is s.p.d. and $I_{K}$ denotes the indicator functional of a closed convex set $K$. In this case, (A1) holds with

$$
\varphi(x)=\frac{1}{2}\langle A x, x\rangle+I_{K},
$$

and $x=F^{-1}(y)$ is the unique solution of the variational inequality

$$
x \in K: \quad\langle A x-y, v-x\rangle \geq 0 \quad \forall v \in K .
$$

It is well-known that the corresponding mapping $F^{-1}:\left(\mathbb{R}^{n},\|\cdot\|_{A^{-1}}\right) \rightarrow\left(\mathbb{R}^{n},\|\cdot\|_{A}\right)$ is Lipschitz continuous with constant $L_{F^{-1}} \leq 1$ (cf., e.g., [29, p. 24]). 
2.2. Nonlinear Schur complement and unconstrained minimization. Our aim is to reformulate the given saddle point problem as an unconstrained minimization problem. In the first step the inclusion (1.1) is transformed into a single-valued equation.

Proposition 2.1. The saddle point problem (1.1) is equivalent to

$$
w^{*} \in \mathbb{R}^{m}: \quad H\left(w^{*}\right)=0
$$

with the Lipschitz continuous mapping

$$
H(w)=-B F^{-1}\left(f-B^{T} w\right)+C w+g, \quad w \in \mathbb{R}^{m} .
$$

Proof. Using (A1) the equivalence is easily obtained by straightforward block elimination. Lipschitz-continuity is clear since $H$ consists of a sum and a composition of the Lipschitz continuous function $F^{-1}$ with linear and constant functions.

The operator $H$ can be regarded as a nonlinear version of the well-known Schur complement. In contrast to the linear case, the right-hand side $f$ cannot be separated from the part depending on $w$. Note, that $H$ is single-valued, because $F^{-1}=(\partial \varphi)^{-1}$ is single-valued or, equivalently, the minimization of $\varphi$ on $\mathbb{R}^{n}$ admits a unique solution.

Theorem 2.1. There is a Fréchet-differentiable, convex functional $h: \mathbb{R}^{m} \rightarrow \mathbb{R}$ with the property $\nabla h=H$ and the representation

$$
h(w)=-\mathcal{L}\left(F^{-1}\left(f-B^{T} w\right), w\right), \quad w \in \mathbb{R}^{m},
$$

where

$$
\mathcal{L}(u, w)=\varphi(u)-\langle f, u\rangle+\langle B u-g, w\rangle-\frac{1}{2}\langle C w, w\rangle
$$

denotes the Lagrange functional associated with (1.1).

Proof. The polar (or conjugate) functional $\varphi^{*}$ of $\varphi$ is convex and, by Corollary 5.2 in $\left[17\right.$, p. 22], has the property $\partial \varphi^{*}=(\partial \varphi)^{-1}=F^{-1}$. Since $F^{-1}$ is singlevalued, $\varphi^{*}$ is Gâteaux-differentiable. The continuity of $F^{-1}$ implies that $\varphi^{*}$ is even Fréchet-differentiable with $\nabla \varphi^{*}=F^{-1}$. Setting

$$
h(w)=\varphi^{*}\left(f-B^{T} w\right)+\frac{1}{2}\langle C w, w\rangle+\langle g, w\rangle
$$

we immediately get $\nabla h=H$ using the chain rule. By definition of $\varphi^{*}$ we have

$$
\begin{aligned}
\varphi^{*}(y) & =\sup _{x \in \mathbb{R}^{n}}(\langle y, x\rangle-\varphi(x))=-\inf _{x \in \mathbb{R}^{n}}(\varphi(x)-\langle y, x\rangle) \\
& =-\left(\varphi\left(F^{-1}(y)\right)-\left\langle y, F^{-1}(y)\right\rangle\right), \quad y \in \mathbb{R}^{n} .
\end{aligned}
$$

Inserting this representation with $y=f-B^{T} w$ into (2.5), we get (2.4).

The convexity of $\varphi$ implies the monotonicity of $F^{-1}$. In combination with the non-negativity of $C$ we get

$$
\begin{aligned}
& \left\langle w_{1}-w_{2}, H\left(w_{1}\right)-H\left(w_{2}\right)\right\rangle \\
& =\left\langle\left(f-B^{T} w_{1}\right)-\left(f-B^{T} w_{2}\right), F^{-1}\left(f-B^{T} w_{1}\right)-F^{-1}\left(f-B^{T} w_{2}\right)\right\rangle \\
& +\left\langle C\left(w_{1}-w_{2}\right), w_{1}-w_{2}\right\rangle \geq 0
\end{aligned}
$$

so that $H$ is monotone. Therefore $h$ is convex. 
Assuming, in addition to (A2), that $C$ is positive definite, it is not difficult to show that $h$ is strongly convex, i.e., there is a constant $\mu>0$ such that

$$
h(\lambda x+(1-\lambda) y) \leq \lambda h(x)+(1-\lambda) h(y)-\lambda(1-\lambda) \frac{\mu}{2}\|x-y\|_{M}^{2} \quad \forall \lambda \in[0,1]
$$

holds for all $x, y \in \mathbb{R}^{m}$. In general, however, $h$ is not even strictly convex so that we had to require uniqueness separately.

Combining Proposition 2.1 with Theorem 2.1, we are ready to state the main result of this section.

Corollary 2.1. The set-valued saddle point problem (1.1) is equivalent to the unconstrained convex minimization problem

$$
w^{*} \in \mathbb{R}^{m}: \quad h\left(w^{*}\right) \leq h(w) \quad \forall w \in \mathbb{R}^{m} .
$$

Recall that the functional $h$ is differentiable with Lipschitz continuous gradient $H=\nabla h$. However, the actual evaluation of $h(w)$ and $\nabla h(w)$ might be expensive, because it involves the solution of $F(u)=f-B^{T} w$.

\section{Gradient-Related methods}

Exploiting Corollary 2.1, existing algorithms for the unconstrained minimization of convex, differentiable functionals now can be utilized to solve the constrained saddle point problem (1.1). In this section, we consider the fairly general class of gradient-related descent methods (see for example [39]). In agreement with Section 2.2, we assume that $h: \mathbb{R}^{m} \rightarrow \mathbb{R}$ denotes a convex functional with Lipschitz continuous Fréchet derivative $\nabla h$ and the unique minimizer $w^{*} \in \mathbb{R}^{m}$.

3.1. Global convergence results. We consider the iteration

$$
w^{\nu+1}=w^{\nu}+\rho^{\nu} d^{\nu}, \quad \nu=0,1, \ldots,
$$

with given initial guess $w^{0} \in \mathbb{R}^{m}$. In each step, first a search direction $d^{\nu}$ is chosen according to the actual iterate $w^{\nu}$ and then a step size $\rho^{\nu}$ is fixed according to $w^{\nu}$ and $d^{\nu}$, i.e.,

$$
d^{\nu}=d\left(\nu, w^{\nu}\right), \quad \rho^{\nu}=\rho\left(\nu, w^{\nu}, d^{\nu}\right), \quad \nu=0,1, \ldots
$$

with suitable mappings $d, \rho$.

The search directions $d^{\nu}$ should allow for a sufficient descent of $h$.

Definition 3.1. The search directions $d^{\nu}=d\left(\nu, w^{\nu}\right), \nu \in \mathbb{N}$, are called gradientrelated descent directions, if for any sequence $\left(w^{\nu}\right) \subset \mathbb{R}^{m}$ the conditions

$$
\nabla h\left(w^{\nu}\right)=0 \Longleftrightarrow d^{\nu}=0 \quad \forall \nu \in \mathbb{N}
$$

and

$$
-\left\langle\nabla h\left(w^{\nu}\right), d^{\nu}\right\rangle \geq c_{D}\left\|\nabla h\left(w^{\nu}\right)\right\|_{M^{-1}}\left\|d^{\nu}\right\|_{M} \quad \forall \nu \in \mathbb{N}
$$

hold with a constant $c_{D}>0$ independent of $\nu$.

Note that the preconditioned gradients $d^{\nu}=-M^{-1} \nabla h\left(w^{\nu}\right)$ satisfy (3.4) with equality and $c_{D}=1$. Obviously, (3.4) implies

$$
-\left(\nabla h\left(w^{\nu}\right), d^{\nu}\right)>0,
$$


if $\nabla h\left(w^{\nu}\right) \neq 0$. Search directions $d^{\nu}=d\left(\nu, w^{\nu}\right), \nu \in \mathbb{N}$, satisfying (3.3) and, instead of (3.4), the weaker condition (3.5) for arbitrary $\left(w^{\nu}\right) \in \mathbb{R}^{m}$ are called descent directions.

The step sizes $\rho^{\nu}$ should realize a sufficient portion of possible descent.

Definition 3.2. Let $d^{\nu}=d\left(\nu, w^{\nu}\right), \nu \in \mathbb{N}$, be descent directions. Then the step sizes $\rho^{\nu}=\rho\left(\nu, w^{\nu}, d^{\nu}\right), \nu \in \mathbb{N}$, are called efficient, if for any sequence $\left(w^{\nu}\right) \subset \mathbb{R}^{m}$ the estimate

$$
h\left(w^{\nu}+\rho^{\nu} d^{\nu}\right) \leq h\left(w^{\nu}\right)-c_{S}\left(\frac{\left\langle\nabla h\left(w^{\nu}\right), d^{\nu}\right\rangle}{\left\|d^{\nu}\right\|_{M}}\right)^{2}
$$

holds for all $\nu \in \mathbb{N}$ such that $\nabla h\left(w^{\nu}\right) \neq 0$ with a constant $c_{S}>0$ independent of $\nu$.

We are now ready to prove convergence.

Theorem 3.1. Assume that (3.2) provides gradient-related descent directions $d^{\nu}$ and efficient step sizes $\rho^{\nu}$. Then, for arbitrary initial iterate $w^{0} \in \mathbb{R}^{m}$, the iterates $w^{\nu}, \nu \in \mathbb{N}$, obtained from (3.1) converge to the minimizer $w^{*}$ of $h$.

Proof. Combining the properties of $d^{\nu}=d\left(\nu, w^{\nu}\right)$ and $\rho^{\nu}=\rho\left(\nu, w^{\nu}, d^{\nu}\right)$ we get

$$
h\left(w^{\nu}\right)-h\left(w^{\nu+1}\right) \geq c_{S} c_{D}^{2}\left\|\nabla h\left(w^{\nu}\right)\right\|_{M^{-1}}^{2} \quad \forall \nu \in \mathbb{N} .
$$

Since $h$ has a global minimizer, the sequence $\left(h\left(w^{\nu}\right)\right)$ is bounded from below and, by (3.7), is monotonically decreasing. Hence, $h\left(w^{\nu}\right)$ converges to some $h^{*} \in \mathbb{R}$. Using again (3.7), we get

$$
0 \leq c_{S} c_{D}^{2}\left\|\nabla h\left(w^{\nu}\right)\right\|_{M^{-1}}^{2} \leq h\left(w^{\nu}\right)-h\left(w^{\nu+1}\right) \rightarrow 0
$$

so that $\nabla h\left(w^{\nu}\right)$ must tend to zero.

The section $S=\left\{w \in \mathbb{R}^{m} \mid h(w) \leq h\left(w^{0}\right)\right\}$ is bounded. Otherwise there would be a sequence $\left(w_{k}\right) \subset S$ with the property $\lambda_{k}^{-1}:=\left\|w_{k}-w^{*}\right\| \geq k$. Then, by compactness of the unit sphere with center $w^{*}$, the sequence $w_{k}^{\prime}=w^{*}+\left(w_{k}-\right.$ $\left.w^{*}\right) /\left\|w_{k}-w^{*}\right\|$ has a convergent subsequence $w_{k_{j}}^{\prime} \rightarrow w^{* *} \neq w^{*}$. By continuity and convexity of $h$ this leads to

$$
h\left(w^{* *}\right)=\lim _{j \rightarrow \infty} h\left(w_{k_{j}}^{\prime}\right) \leq \lim _{j \rightarrow \infty} \lambda_{k_{j}} h\left(w_{k_{j}}\right)+\left(1-\lambda_{k_{j}}\right) h\left(w^{*}\right)=h\left(w^{*}\right),
$$

contradicting the uniqueness of $w^{*}$.

The section $S$ is also closed and therefore compact. As a consequence, $\left(w^{\nu}\right)$ has a convergent subsequence $\left(w^{\nu_{i}}\right) \rightarrow w^{* *}$. The continuity of $\nabla h$ provides $\nabla h\left(w^{* *}\right)=0$ and uniqueness implies $w^{* *}=w^{*}$. Hence, each convergent subsequence must tend to $w^{*}$. This proves the assertion.

In the proof, we have made extensive use of Heine-Borel's Theorem which is restricted to finite dimensions. However, using weak compactness and the weak lower semi-continuity of $h$, weak convergence of the iterates $w^{\nu}$ can be shown by similar arguments in the infinite-dimensional case. Strong linear convergence can be shown in any dimension under the additional assumption that $h$ is strongly convex. The proof is based on the following lemma summarizing well known results (cf., e.g., [39]). 
Lemma 3.1. Let $h$ be strongly convex with constant $\mu>0$. Then $h$ satisfies the estimates

$$
\frac{\mu}{2}\left\|w-w^{*}\right\|_{M}^{2} \leq h(w)-h\left(w^{*}\right) \leq \frac{1}{2 \mu}\|\nabla h(w)\|_{M^{-1}}^{2} \quad \forall w \in \mathbb{R}^{m}
$$

with the minimizer $w^{*}$ of $h$.

Theorem 3.2. Assume that the conditions of Theorem 3.1 are satisfied and, in addition, $h$ is strongly convex with constant $\mu>0$. Then the iterates $w^{\nu}, \nu \in \mathbb{N}$, produced by (3.1) satisfy the error estimate

$$
\left\|w^{\nu}-w^{*}\right\|_{M}^{2} \leq q^{\nu} \frac{2}{\mu}\left(h\left(w^{0}\right)-h\left(w^{*}\right)\right)
$$

where $0 \leq q=\left(1-2 c_{S} c_{D}^{2} \mu\right)<1$ if $w^{0} \neq w^{*}$.

The proof is straightforward using Lemma 3.1.

3.2. Damping strategies. A variety of algorithms for efficient step size control is available from surveys and textbooks like [16, 38, 39, 40]. For simplicity, we consider the standard Armijo strategy [2], [16, p. 121], [39, p. 491] based on the actual decrease of the functional $h$. More precisely, for a fixed parameter $\delta \in(0,1)$ and each $\nu \in \mathbb{N}$ a step size $\rho \geq 0$ is called admissible, if

$$
h\left(w^{\nu}+\rho d^{\nu}\right) \leq h\left(w^{\nu}\right)+\rho \delta\left\langle\nabla h\left(w^{\nu}\right), d^{\nu}\right\rangle
$$

is satisfied.

Proposition 3.1. Let $\left(w^{\nu}\right) \subset \mathbb{R}^{m}$ and let $d^{\nu}=d\left(\nu, w^{\nu}\right), \nu \in \mathbb{N}$, be descent directions. For suitably selected, fixed parameters $\alpha>0$ and $\delta, \beta \in(0,1)$ determine the step sizes $\rho^{\nu}=\rho\left(\nu, w^{\nu}, d^{\nu}\right) \geq 0$ by

$$
\rho^{\nu}=\max _{j \in \mathbb{N} \cup\{0\}}\left\{\rho=\alpha_{\nu} \beta^{j} \mid \alpha_{\nu} \geq-\alpha \frac{\left\langle\nabla h\left(w^{\nu}\right), d^{\nu}\right\rangle}{\left\|d^{\nu}\right\|_{M}^{2}}, \rho \text { admissible }\right\}
$$

if $d^{\nu} \neq 0$ and set $\rho^{\nu}=0$ otherwise. Then the efficiency condition (3.6) holds with

$$
c_{S}=\delta \min \left\{\alpha, \beta\left(\frac{1-\delta}{L}\right)\right\} .
$$

Here $L$ stands for the Lipschitz constant of $\nabla h$, i.e.,

$$
\|\nabla h(v)-\nabla h(w)\|_{M^{-1}} \leq L\|v-w\|_{M} \quad \forall v, w \in \mathbb{R}^{m} .
$$

The proof of Proposition 3.1 adopts standard arguments, e.g., from [39]. Starting with $j=0$, efficient step sizes can be computed from (3.12) by a finite number of tests. Observe that each of these tests might be expensive, because it requires the evaluation of $h$ and therefore the evaluation of $F^{-1}$ (cf. Theorem 2.1).

3.3. Inexact versions. We consider inexact search directions $\tilde{d}^{\nu}$. This means that for given $\nu$ and $w^{\nu}$ the exact evaluation $d^{\nu}=d\left(\nu, w^{\nu}\right)$ is replaced by some approximation

$$
\tilde{d}^{\nu}=\tilde{d}\left(\nu, w^{\nu}\right)
$$

based on some approximation $\tilde{d}$ of the exact mapping $d$. 
Proposition 3.2. Let $d^{\nu}=d\left(\nu, w^{\nu}\right)$ be gradient-related descent directions with constant $c_{D}$. Assume that the approximations $\tilde{d}^{\nu}=\tilde{d}\left(\nu, w^{\nu}\right)$ satisfy (3.3) and the accuracy condition

$$
\frac{\left\|d^{\nu}-\tilde{d}^{\nu}\right\|_{M}}{\left\|\tilde{d}^{\nu}\right\|_{M}} \leq c<\frac{c_{D}}{2} \quad \forall \nu \in \mathbb{N}
$$

for any sequence $\left(w^{\nu}\right)$. Then the approximations $\tilde{d}^{\nu}=\tilde{d}\left(\nu, w^{\nu}\right)$ are also gradientrelated descent directions.

Proof. Let $\left(w^{\nu}\right) \subset \mathbb{R}^{m}$. Then, the vectors $d^{\nu}=d\left(\nu, w^{\nu}\right), \nu \in \mathbb{N}$, satisfy (3.4) and we have to prove a similar estimate for the approximations $\tilde{d}^{\nu}$. This is trivial for $\tilde{d}^{\nu}=0$. In the light of (3.3) there is only the remaining case $d^{\nu}, \tilde{d}^{\nu} \neq 0$. Some elementary calculations involving the Cauchy-Schwarz inequality and the triangle inequality yield

$$
\left|\left\langle\frac{\nabla h\left(w^{\nu}\right)}{\left\|\nabla h\left(w^{\nu}\right)\right\|_{M^{-1}}}, \frac{d^{\nu}}{\left\|d^{\nu}\right\|_{M}}-\frac{\tilde{d}^{\nu}}{\left\|\tilde{d}^{\nu}\right\|_{M}}\right\rangle\right| \leq 2 \frac{\left\|d^{\nu}-\tilde{d}^{\nu}\right\|_{M}}{\left\|\tilde{d}^{\nu}\right\|_{M}} .
$$

As $\left\|d^{\nu}-\tilde{d}^{\nu}\right\|_{M} /\left\|\tilde{d}^{\nu}\right\|_{M} \leq c<c_{D} / 2$, it is clear that

$$
-\left\langle\nabla h\left(w^{\nu}\right), \tilde{d}^{\nu}\right\rangle \geq \tilde{c}_{D}\left\|\nabla h\left(w^{\nu}\right)\right\|_{M^{-1}}\left\|\tilde{d}^{\nu}\right\|_{M}
$$

with $\tilde{c}_{D}=c_{D}-2 c>0$.

Usually the constant $c_{D}$ occurring in the accuracy condition (3.16) is not known. Replacing (3.16) by the asymptotic criterion

$$
\lim _{\nu \rightarrow \infty} \frac{\left\|d^{\nu}-\tilde{d}^{\nu}\right\|_{M}}{\left\|\tilde{d}^{\nu}\right\|_{M}}=0
$$

the approximate directions $\tilde{d}^{\nu}$ have the desired property (3.4) for sufficiently large $\nu$.

\section{Nonsmooth Newton methods AND Related Algorithms}

We now consider the question how to choose the descent directions $d^{\nu}=d\left(w^{\nu}\right)$. We will concentrate on preconditioned gradients of $h$ or, more precisely, on directions of the form

$$
d^{\nu}=-S_{\nu}^{-1} H\left(w^{\nu}\right), \quad H=\nabla h,
$$

with suitable s.p.d. matrices $S_{\nu}=S\left(\nu, w^{\nu}\right)$. If $H$ would be sufficiently smooth, the derivative

$$
S_{\nu}=H^{\prime}\left(w^{\nu}\right): \mathbb{R}^{m} \rightarrow \mathbb{R}^{m}
$$

would provide the classical Newton iteration. From our assumptions (A1) - (A4) and the definition (2.3), we cannot expect $H^{\prime}$ to exist. Hence, related concepts from nonsmooth analysis will be applied. To this end, (A1) is from now on replaced by the stronger condition (A1'):

(A1') $F=A+\partial I_{K}$ where $A \in \mathbb{R}^{n, n}$ is s.p.d. and $I_{K}$ denotes the indicator functional of the closed convex set

$$
K=\left\{x \in \mathbb{R}^{n} \mid a \leq x \leq b\right\} \quad a, b \in(\mathbb{R} \cup\{-\infty, \infty\})^{n}, \quad a<0<b .
$$


Recall that $F$ is the subdifferential of $\varphi(x)=\frac{1}{2}\langle A x, x\rangle+I_{K}$ and Lipschitz continuous with constant $L \leq 1$ in this case. Nonlinearities $F$ satisfying (A1') occur, e.g., in discretized optimal control problems with inequality constraints $[34,47]$ or discretized phase field models with obstacle potentials [6,8]. The condition $a<0<b$ causes no loss of generality and will be notationally convenient in the sequel.

4.1. The B-subdifferential of $F^{-1}$. Let $c \in K$ with $K \subset \mathbb{R}^{n}$ defined in (4.2). We introduce the subset of all active indices

$$
N_{c}^{\bullet}:=\left\{i \in N \mid a_{i}=c_{i} \text { or } c_{i}=b_{i}\right\}
$$

of the index set $N=\{1, \ldots, n\}$. The mapping $T_{c}: \mathbb{R}^{n} \rightarrow \mathbb{R}^{n}$, defined by

$$
T_{c} x:=\sum_{i \in N \backslash N_{c}^{\bullet}} x_{i} e_{i}, \quad x \in \mathbb{R}^{n},
$$

truncates all coefficients with active indices. Note that $T_{c}$ is an orthogonal projection with respect to the euclidian scalar product $\langle\cdot, \cdot\rangle$. The finite set

$$
\mathcal{C}:=\left\{c \in K \mid\left(I-T_{c}\right) c=c\right\}
$$

represents all possible configurations of active coefficients, i.e., of coefficients with active indices. The active coefficients of $x \in K$ are given by

$$
T_{\mathcal{C}} x:=\left(I-T_{x}\right) x \in \mathcal{C} .
$$

As $F: K \rightarrow \mathbb{R}^{n}$ is invertible, $K$ and $\mathbb{R}^{n}$ can be decomposed according to

$$
K=\bigcup_{c \in \mathcal{C}} \mathcal{I}_{c}, \quad \mathbb{R}^{n}=\bigcup_{c \in \mathcal{C}} F\left(\mathcal{I}_{c}\right), \quad \mathcal{I}_{c}:=\left\{x \in K \mid T_{\mathcal{C}} x=c\right\},
$$

based on the subsets $\mathcal{I}_{c}$ of vectors with the same active coefficients. Note that

$$
\left(I-T_{c}\right) x=c \quad \forall x \in \mathcal{I}_{c}, \quad c \in \mathcal{C} .
$$

We now investigate the restriction of $F$ to $\mathcal{I}_{c}$. To this end, it is convenient to introduce the mapping

$$
\widehat{A}_{c}:=T_{c} A T_{c}+I-T_{c}: \mathbb{R}^{n} \rightarrow \mathbb{R}^{n} .
$$

Observe that $\widehat{A}_{c}: \operatorname{ran} T_{c} \rightarrow \operatorname{ran} T_{c}$ and $\widehat{A}_{c}$ reduces to the identity on the orthogonal complement $\operatorname{ran}\left(I-T_{c}\right)$. Hence,

$$
\widehat{A}_{c} T_{c}=T_{c} A T_{c}=T_{c} \widehat{A}_{c}, \quad \widehat{A}_{c}\left(I-T_{c}\right)=I-T_{c} .
$$

Using

$$
\left\langle\widehat{A}_{c} x, y\right\rangle=\left\langle A T_{c} x, T_{c} y\right\rangle+\left\langle\left(I-T_{c}\right) x,\left(I-T_{c}\right) y\right\rangle
$$

it is easy to show that $\widehat{A}_{c}$ is s.p.d. Multiplying (4.6) with $\widehat{A}_{c}^{-1}$ we obtain

$$
\widehat{A}_{c}^{-1} T_{c}=T_{c} \widehat{A}_{c}^{-1}, \quad \widehat{A}_{c}^{-1}\left(I-T_{c}\right)=I-T_{c} .
$$

Lemma 4.1. Let $c \in \mathcal{C}$. Then the restriction of $F$ to $\mathcal{I}_{c}$ takes the form

$$
F(x)=A x+\sum_{i \in N_{c}}[0, \infty) s_{i}(c) e_{i}, \quad x \in \mathcal{I}_{c},
$$

denoting

$$
s_{i}(c)=\left\{\begin{array}{ll}
+1 & \text { if } c_{i}=b_{i} \\
-1 & \text { if } c_{i}=a_{i}
\end{array}, \quad i \in N_{c}^{\bullet}\right.
$$


Conversely, the restriction of $F^{-1}$ to $F\left(\mathcal{I}_{c}\right)$ takes the form

$$
F^{-1}(y)=T_{c} \widehat{A}_{c}^{-1} T_{c} y+\left(I-T_{c} \widehat{A}_{c}^{-1} T_{c} A\right) c, \quad y \in F\left(\mathcal{I}_{c}\right) .
$$

Proof. Let $x \in \mathcal{I}_{c}$. Using the representation

$$
I_{K}(x)=\sum_{i \in N} I_{\left[a_{i}, b_{i}\right]}\left(x_{i}\right), \quad x=\sum_{i \in N} x_{i} e_{i},
$$

of the characteristic functional $I_{K}$, we immediately get (cf. [17, page 26])

$$
\partial I_{K}(x)=\sum_{i \in N} \partial I_{\left[a_{i}, b_{i}\right]}\left(x_{i}\right) e_{i}=\sum_{i \in N_{c}^{\bullet}}[0, \infty) s_{i}(c) e_{i}
$$

This proves (4.8).

Let $x \in \mathcal{I}_{c}$ and $y \in F(x)$. We apply $T_{c}$ to the representation (4.8), insert the splitting $x=T_{c} x+\left(I-T_{c}\right) x$, and use the identity $\left(I-T_{c}\right) x=c$ to obtain

$$
T_{c} y=T_{c} A x=T_{c} A T_{c} x+T_{c} A c=\widehat{A}_{c} x-\left(I-T_{c} A\right) c .
$$

Multiplication by $\widehat{A}_{c}^{-1}$ and reordering terms, we get

$$
x=\widehat{A}_{c}^{-1} T_{c} y+\widehat{A}_{c}^{-1}\left(I-T_{c} A\right) c .
$$

The left identity in (4.7) yields

$$
\widehat{A}_{c}^{-1} T_{c}=\widehat{A}_{c}^{-1} T_{c} T_{c}=T_{c} \widehat{A}_{c}^{-1} T_{c} .
$$

Using $c=\left(I-T_{c}\right) c$ and the right identity in (4.7), we obtain

$$
\widehat{A}_{c}^{-1} c=\widehat{A}_{c}^{-1}\left(I-T_{c}\right) c=\left(I-T_{c}\right) c=c .
$$

Inserting these representations into (4.10) the assertion (4.9) follows.

As a consequence of (4.4) and (4.9), $F^{-1}$ is piecewise affine linear on $\mathbb{R}^{n}$ with the linear part $T_{c} \widehat{A}_{c}^{-1} T_{c}$ on each subset $F\left(\mathcal{I}_{c}\right), c \in \mathcal{C}$. In the extreme case, $N_{c}^{\bullet}=N$, $F^{-1}$ is even constant on $F\left(\mathcal{I}_{c}\right)$.

As $F^{-1}$ is Lipschitz continuous, $F^{-1}$ must be differentiable almost everywhere (cf. Rademacher's theorem [37]). Let $\mathcal{D}_{F^{-1}}$ denote the set where $F^{-1}$ is differentiable. Then the B-subdifferential $\partial_{B}\left(F^{-1}\right)$ (cf. $\left.[41,48]\right)$ is defined by

$$
\partial_{B}\left(F^{-1}\right)(y)=\left\{\lim _{\substack{y_{n} \rightarrow y \\ y_{n} \in \mathcal{D}_{F^{-1}}}} D\left(F^{-1}\right)\left(y_{n}\right)\right\} .
$$

Note that

$$
\partial_{B}\left(F^{-1}\right)(y) \subset \operatorname{co} \partial_{B}\left(F^{-1}\right)(y)=\partial\left(F^{-1}\right)(y)
$$

with $\partial\left(F^{-1}\right)$ denoting Clarke's generalized derivative [13, Chapter 2].

Proposition 4.1. Let $y \in \mathbb{R}^{n}$ and $c=T_{\mathcal{C}}\left(F^{-1}(y)\right) \in \mathcal{C}$. Then

$$
T_{c} \widehat{A}_{c}^{-1} T_{c} \in \partial_{B}\left(F^{-1}\right)(y) \text {. }
$$

Proof. Note that $F^{-1}(y) \in \mathcal{I}_{c}$ by definition (4.4) of $\mathcal{I}_{c}$. Inserting the decomposition $x=T_{c} x+c$ of some arbitrary $x \in \mathcal{I}_{c}$ into (4.8), it turns out that $F\left(\mathcal{I}_{c}\right)$ is the parallelepiped translated from the origin by $A c$ and spanned by the nonzero column vectors of $A T_{c}$ and of $I-T_{c}$ with coefficients $z_{i} \in\left(a_{i}, b_{i}\right), i \in N \backslash N_{c}^{\bullet}$, and $z_{i} \in$ 


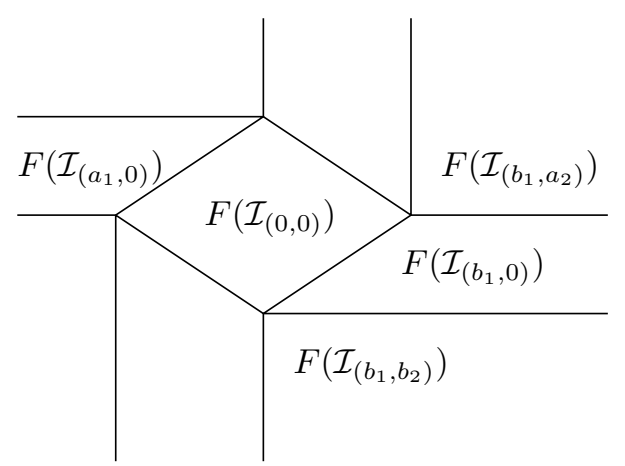

Figure 4.1. Decomposition of $\mathbb{R}^{2}$ into parallelepipeds $F\left(\mathcal{I}_{c}\right), c \in \mathcal{C}$.

$[0, \infty) s_{i}(c), i \in N_{c}^{\bullet}$, respectively. Utilizing the identities $A T_{c}+I-T_{c}=\widehat{A}_{c}+(I-$ $\left.T_{c}\right) A T_{c},(4.7)$, and the orthogonality $T_{c}\left(I-T_{c}\right)=0$, it is easily checked that

$$
\left(\widehat{A}_{c}^{-1}-\left(I-T_{c}\right) A T_{c} \widehat{A}_{c}^{-1}\right)\left(A T_{c}+I-T_{c}\right)=I .
$$

Hence, the interior of $F\left(\mathcal{I}_{c}\right)$ cannot be empty so that the convexity of $F\left(\mathcal{I}_{c}\right)$ yields

$$
F\left(\mathcal{I}_{c}\right) \subset \overline{\operatorname{int} F\left(\mathcal{I}_{c}\right)} .
$$

If $y \in \operatorname{int} F\left(\mathcal{I}_{c}\right)$, then the representation (4.9) implies

$$
D\left(F^{-1}\right)(y)=T_{c} \widehat{A}_{c}^{-1} T_{c} .
$$

If $y \in F\left(\mathcal{I}_{c}\right) \backslash \operatorname{int} F\left(\mathcal{I}_{c}\right)$, then (4.12) implies that there is a sequence $\left(y_{k}\right) \subset \operatorname{int} F\left(\mathcal{I}_{c}\right)$ with $y_{k} \rightarrow y$. Obviously,

$$
\lim _{k \rightarrow \infty} D F^{-1}\left(y_{k}\right)=T_{c} \widehat{A}_{c}^{-1} T_{c}
$$

which proves the assertion.

Figure 4.1 illustrates the decomposition of $\mathbb{R}^{n}$ into the nondegenerating parallelepipeds $F\left(\mathcal{I}_{c}\right), c \in \mathcal{C}$, for $n=2$. The only bounded parallelepiped $F\left(\mathcal{I}_{(0,0)}\right)$ is spanned by the column vectors of $A$.

4.2. Algorithms and convergence results. Proposition 4.1 suggests to use Bsubdifferentials $T_{c} \widehat{A}_{c}^{-1} T_{c}, c \in \mathcal{C}$, for the linearization of the Schur complement

$$
H(w)=-B F^{-1}\left(f-B^{T} w\right)+C w+g, \quad w \in \mathbb{R}^{m},
$$

as introduced in (2.3).

Proposition 4.2. Assume that $\operatorname{rank} B=n$. Then

$$
S(c)=B T_{c} \widehat{A}_{c}^{-1}\left(B T_{c}\right)^{T}+C \in \partial_{B} H(w), \quad w \in \mathbb{R}^{m},
$$

where

$$
c=c(w)=T_{\mathcal{C}} F^{-1}\left(f-B^{T} w\right) .
$$


Proof. Let $G: \mathbb{R}^{m} \rightarrow \mathbb{R}^{n}$ be defined by $G(w)=F^{-1}\left(f-B^{T} w\right), w \in \mathbb{R}^{m}$. We consider some fixed $w \in \mathbb{R}^{m}$ and $c=T_{\mathcal{C}} G(w)$. As rank $B=n$, the mapping $B^{T}: \mathbb{R}^{m} \rightarrow \mathbb{R}^{n}$ is surjective. Hence, the preimage $G^{-1}\left(\mathcal{I}_{c}\right)$ of $\mathcal{I}_{c}$ is still a nondegenerate parallelepiped. Therefore we can use the same arguments as in the proof of Proposition 4.1 to show

$$
-T_{c} \widehat{A}_{c}^{-1} T_{c} B^{T} \in \partial_{B} G(w) .
$$

As $H$ is an affine transformation of $G$, the assertion follows.

Simple counterexamples show that (4.13) might not hold for $\operatorname{rank} B^{T}<n$.

Let us check whether $S(c)$ is invertible. We immediately get

$$
\langle S(c) x, y\rangle=\left\langle\widehat{A}_{c}^{-1}\left(B T_{c}\right)^{T} x,\left(B T_{c}\right)^{T} y\right\rangle+\langle C x, y\rangle, \quad x, y \in \mathbb{R}^{m} .
$$

Hence, $S(c)$ is symmetric and positive semi-definite. It is a sufficient (but not necessary) condition for the regularity of $S(c)$ that $C$ is s.p.d..

Lemma 4.2. Assume that $S(c)$ is s.p.d. for all $c \in \mathcal{C}$. Then $h$ is strongly convex.

Proof. Consider $G(w)=F^{-1}\left(f-B^{T} w\right)$ as already introduced in the proof of Proposition 4.2. Let $c \in \mathcal{C}$. Then, for all $w \in G^{-1} \mathcal{I}_{c}$ the representation $\nabla h(w)=$ $H(w)=S(c) w+\tilde{g}(c)$ holds with suitable $\tilde{g}(c) \in \mathbb{R}^{m}$ independent of $w$ (cf. Lemma 4.1). As $S(c)$ is s.p.d., we have

$$
\langle S(c) w, w\rangle \geq \gamma_{c}\|w\|_{M}^{2} \quad \forall w \in G^{-1} \mathcal{I}_{c}
$$

with some constant $\gamma_{c}>0$. This means that $h$ is quadratic and strongly convex on each preimage $G^{-1} \mathcal{I}_{c}$. We now show strong convexity on the whole $\mathbb{R}^{m}=$ $\bigcup_{c \in \mathcal{C}} G^{-1} \mathcal{I}_{c}$ with the constant $\mu=\min _{c \in \mathcal{C}} \gamma_{c}>0$. To this end, we define the scalar functions

$$
\begin{aligned}
& \psi_{1}(\lambda)=\|x-y\|_{M}^{-2} h(\lambda x+(1-\lambda) y) \\
& \psi_{2}(\lambda)=\|x-y\|_{M}^{-2}(\lambda h(x)+(1-\lambda) h(y))-\frac{\mu}{2} \lambda(1-\lambda)
\end{aligned}, \quad \lambda \in[0,1],
$$

with some fixed $x \neq y \in \mathbb{R}^{m}$. It is sufficient to show $\psi_{1} \leq \psi_{2}$. Obviously, $\psi_{1}$ is piecewise quadratic, $\psi_{2}$ is quadratic, and $\psi_{1}(\lambda)=\psi_{2}(\lambda)$ at the boundary $\lambda=0,1$. By definition,

$$
\psi_{1}^{\prime \prime}(\lambda) \geq \min _{c \in \mathcal{C}} \gamma_{c}=\psi_{2}^{\prime \prime}(\lambda)
$$

holds for almost all $\lambda \in[0,1]$. Now $\psi_{1} \leq \psi_{2}$ follows either from elementary arguments or from a weak maximum principle (cf. [20, Theorem 9.1]) as applied to $\psi_{1}-\psi_{2}$.

We are ready to state the basic convergence result of this section.

Theorem 4.1. Assume that $S(c)$ is s.p.d. for all $c \in \mathcal{C}$. Then, for arbitrary initial iterate $w^{0} \in \mathbb{R}^{m}$, the damped nonsmooth Newton-type method, as obtained by inserting the search directions

$$
d^{\nu}=-S_{\nu}^{-1} H\left(w^{\nu}\right), \quad H\left(w^{\nu}\right)=\nabla h\left(w^{\nu}\right),
$$

with

$$
S_{\nu}=S\left(c^{\nu}\right), \quad c^{\nu}=T_{\mathcal{C}} F^{-1}\left(f-B^{T} w^{\nu}\right)
$$


and step sizes $\rho^{\nu}$ selected according to Proposition 3.1 into the basic algorithm (3.1), converges linearly to the solution $w^{*}$ of (2.8). If (2.8) is nondegenerate in the sense that

$$
F^{-1}\left(f-B^{T} w^{*}\right) \in \operatorname{int} \mathcal{I}_{c^{*}}, \quad c^{*}=T_{\mathcal{C}} F^{-1}\left(f-B^{T} w^{*}\right),
$$

then the algorithm terminates after a finite number of steps.

Proof. To prove convergence by Theorem 3.1, we only have to show that the directions $d^{\nu}$ as defined in (4.16) are gradient-related. Let $c \in \mathcal{C}$. Denoting the norm of the linear mapping $S(c):\left(\mathbb{R}^{m},\|\cdot\|_{M}\right)$ to $\left(\mathbb{R}^{m},\|\cdot\|_{M^{-1}}\right)$ by $\Gamma_{c}$ and using the coercivity (4.15), we get

$\left\langle\nabla h(w), S(c)^{-1} \nabla h(w)\right\rangle \geq \gamma_{c}\left\|S(c)^{-1} \nabla h(w)\right\|_{M}^{2} \geq \frac{\gamma_{c}}{\Gamma_{c}}\left\|S(c)^{-1} \nabla h(w)\right\|_{M}\|\nabla h(w)\|_{M^{-1}}$ for all $w \in \mathbb{R}^{m}$. Since $\mathcal{C}$ is finite, (3.4) now holds with

$$
c_{D}:=\min _{c \in \mathcal{C}} \frac{\gamma_{c}}{\Gamma_{c}}>0 \text {. }
$$

Utilizing Lemma 4.2, linear convergence immediately follows from Theorem 3.2. If (2.8) is nondegenerate, then $F^{-1}\left(f-B^{T} w^{\nu_{0}}\right) \in \mathcal{I}_{c^{*}}$ holds for sufficiently large $\nu_{0}$. This implies $w^{\nu_{0}+1}=w^{*}$, because $H$ is affine on all $w$ with $F^{-1}\left(f-B^{T} w\right) \in \mathcal{I}_{c^{*}}$.

On the additional assumption $\operatorname{rank} B=n$ we obtain (cf. Proposition 4.2)

$$
S_{\nu}=S\left(c^{\nu}\right) \in \partial_{B} H(w) \quad \forall \nu \in \mathbb{N},
$$

and therefore a nonsmooth Newton method. In order to prove local quadratic convergence (cf. [42, 41]), it is essential that $\rho^{\nu} \rightarrow 1$ for $\nu \rightarrow \infty$ which, in general, does not hold for the standard Armijo strategy. Hence, nonsmooth analogues of well-known affine-invariant damping strategies [16, Section 3.4] will be the subject of future research.

If $h$ is not strongly convex, then $S(c)$ is not invertible for certain $c$. Therefore, we now modify $S(c)$ to ensure invertibility.

By symmetry we have $\operatorname{ker} S(c)=(\operatorname{ran} S(c))^{\perp}$. We introduce the mapping $I(c)$ : $\mathbb{R}^{m} \rightarrow \mathbb{R}^{m}$ by

$$
\left.I(c)\right|_{\operatorname{ker} S(c)}=\left.I\right|_{\operatorname{ker} S(c)},\left.\quad I(c)\right|_{\operatorname{ran} S(c)}=0
$$

to define

$$
\widehat{S}(c)=S(c)+I(c), \quad c \in \mathcal{C} .
$$

Observe that the orthogonal subspaces $\operatorname{ker} S(c)$ and $\operatorname{ran} S(c)$ are invariant with respect to $\widehat{S}(c)$. Decomposing $x, y$ into their components from $\operatorname{ker} S(c)$ and $\operatorname{ran} S(c)$, respectively, we get

$$
\langle\widehat{S}(c) x, y\rangle=\left\langle S(c) x_{\mathrm{ran}}, y_{\mathrm{ran}}\right\rangle+\left\langle x_{\mathrm{ker}}, y_{\mathrm{ker}}\right\rangle
$$

so that $\widehat{S}(c)$ is s.p.d. Note that $\widehat{S}(c)$ can be rewritten as

$$
\widehat{S}(c)=S(c)+\sum_{i=1}^{l} \frac{k_{i} k_{i}^{T}}{\left\|k_{i}\right\|^{2}}
$$

with $k_{1}, \ldots, k_{l}$ denoting an orthogonal basis of $\operatorname{ker} S(c)$. If $S(c)$ is replaced by $\widehat{S}(c)$, then nonsmooth Newton steps are carried out on $\operatorname{ran} S_{\nu}$, i.e., if possible, while simple gradient steps are performed on ker $S_{\nu}$. 
Theorem 4.2. For arbitrary initial iterate $w^{0} \in \mathbb{R}^{m}$, the nonsmooth Newton-like method, as obtained by inserting the search directions

$$
d^{\nu}=-\widehat{S}_{\nu}^{-1} H\left(w^{\nu}\right), \quad H\left(w^{\nu}\right)=\nabla h\left(w^{\nu}\right),
$$

with

$$
\widehat{S}_{\nu}=\widehat{S}\left(c^{\nu}\right), \quad c^{\nu}=T_{\mathcal{C}} F^{-1}\left(f-B^{T} w^{\nu}\right)
$$

and step sizes $\rho^{\nu}$ selected according to Proposition 3.1 into the basic algorithm (3.1), converges to the solution $w^{*}$ of (2.8). If the problem (2.8) is nondegenerate in the sense of (4.17) and $S\left(c^{*}\right), c^{*}=T_{\mathcal{C}} F^{-1}\left(f-B^{T} w^{*}\right)$, is positive definite, then the algorithm terminates after a finite number of steps.

Proof. Using the same arguments as in the proof of Theorem 4.1 it can shown that the modified search directions $d^{\nu}$ defined in (4.20) are gradient-related. Hence, convergence is a consequence of Theorem 3.1. Finite termination also follows by the reasoning as in the proof of Theorem 4.1.

Remark . In general one would expect local superlinear convergence of a Newtonlike method. Indeed, this is an immediate consequence of the fact that, in a sufficiently small neighborhood, the algorithms terminate with the exact solution after one step, because $H$ is piecewise affine. Further insight would be obtained by showing that the domain of superlinear convergence is larger than the domain of one step termination and, in particular, does not depend on the dimension $m$.

In order to determine $d^{\nu}=-\widehat{S}_{\nu}^{-1} H\left(w^{\nu}\right)$, a linear saddle point problem associated with the Schur complement matrix $\widehat{S}_{\nu}=\widehat{S}\left(c^{\nu}\right)$ has to be solved (see Section 5 below). Sufficiently accurate iterative solution preserves convergence.

Theorem 4.3. For arbitrary initial iterate $w^{0} \in \mathbb{R}^{m}$, the inexact nonsmooth Newton-like method, as obtained by inserting search directions $\tilde{d}^{\nu}$ which satisfy (3.3) and the accuracy condition (3.16) with $d^{\nu}=-\widehat{S}_{\nu}^{-1} H\left(w^{\nu}\right)$ and step sizes $\rho^{\nu}$ selected according to Proposition 3.1 into the basic algorithm (3.1), converges to the solution $w^{*}$ of (2.8). The iterates converge linearly, if $h$ is strongly convex, e.g., for positive definite $C$.

Proof. As the directions $d^{\nu}$ are gradient related (see the proof of Theorem 4.2 above) the convergence is an immediate consequence of Proposition 3.2. If $C$ is positive definite, then $h$ is strongly convex. In this case linear convergence follows from Theorem 3.2.

\section{Computational Aspects}

5.1. Preconditioned Uzawa methods. Denoting $u^{\nu}:=F^{-1}\left(f-B^{T} w^{\nu}\right)$ the Newton-like method as introduced in Theorem 4.2 can be interpreted as the preconditioned Uzawa iteration

$$
\begin{aligned}
u^{\nu} & =F^{-1}\left(f-B^{T} w^{\nu}\right) \\
w^{\nu+1} & =w^{\nu}+\rho^{\nu} \widehat{S}_{\nu}^{-1}\left(B u^{\nu}-C w^{\nu}-g\right)
\end{aligned}
$$

for the saddle point problem (1.1).

The first substep (5.1a) amounts to the solution of the quadratic obstacle problem

$$
u^{\nu}=\arg \min _{v \in K}\left(\frac{1}{2}\langle A v, v\rangle-\left\langle f-B^{T} w^{\nu}, v\right\rangle\right),
$$


which has been extensively treated in the literature (cf., e.g., [14, 22, 31, 36, 46, 4]).

Inserting the definitions (4.19) and (4.13) of $\widehat{S}_{\nu}$ and $S\left(c^{\nu}\right)$, the evaluation of the preconditioned residual

$$
d^{\nu}=\widehat{S}_{\nu}^{-1}\left(B u^{\nu}-C w^{\nu}-g\right)
$$

in the second substep (5.1b) can be rewritten as the solution of the linear saddle point problem

$$
\left(\begin{array}{cc}
\widehat{A}_{c^{\nu}} & \left(B T_{c^{\nu}}\right)^{T} \\
\left(B T_{c^{\nu}}\right) & -\left(C+I\left(c^{\nu}\right)\right)
\end{array}\right)\left(\begin{array}{l}
\tilde{u}^{\nu} \\
d^{\nu}
\end{array}\right)=\left(\begin{array}{c}
0 \\
g+C w^{\nu}-B u^{\nu}
\end{array}\right) .
$$

where, according to (4.3), $c^{\nu}=T_{\mathcal{C}} u^{\nu}$ identifies the active coefficients of $u^{\nu}$. Recall that $\widehat{A}_{c^{\nu}}$ is obtained from $A$ by replacing the $i$-th row and the $i$-th column by the unit vector $e_{i}$, if $i$ is active, i.e., $c_{i} \in\left\{a_{i}, b_{i}\right\}$. B $T_{c^{\nu}}$ is obtained from $B$ by annihilating the $i$-th column, if $i$ is active. Finally, $I\left(c^{\nu}\right)$ has been defined in (4.18). Thus the preconditioner $\widehat{S}_{\nu}$ is approximating the original set-valued operator by essentially eliminating the actual active coefficients [24]. Sufficiently accurate, iterative solution of (5.3) preserves convergence of the overall iteration (5.1) (cf. Theorem 4.3). In particular, multigrid methods have been investigated in $[9,44,49,54,55]$.

5.2. Inexact evaluation of $F^{-1}$. The exact solution $u^{\nu}=F^{-1}\left(f-B^{T} w^{\nu}\right)$ appears on the right hand side of the linear saddle point problem (5.3). However, it turns out that the preconditioned residual can be computed from $w^{\nu}$ and the active coefficients $c^{\nu}$ of $u^{\nu}$ alone.

Proposition 5.1. For given $w^{\nu} \in \mathbb{R}^{m}$ and $c^{\nu}=T_{\mathcal{C}} u^{\nu}$ let $\left(\tilde{u}^{\nu}, \tilde{w}^{\nu}\right)$ be the solution of

$$
\left(\begin{array}{cc}
\widehat{A}_{c^{\nu}} & \left(B T_{c^{\nu}}\right)^{T} \\
\left(B T_{c^{\nu}}\right) & -\left(C+I\left(c^{\nu}\right)\right)
\end{array}\right)\left(\begin{array}{c}
\tilde{u}^{\nu} \\
\tilde{w}^{\nu}
\end{array}\right)=\left(\begin{array}{c}
T_{c^{\nu}} f-T_{c^{\nu}} A c^{\nu} \\
g-B c^{\nu}-I\left(c^{\nu}\right) w^{\nu}
\end{array}\right) .
$$

Then

$$
\widehat{S}_{\nu}^{-1}\left(B u^{\nu}-C w^{\nu}-g\right)=\tilde{w}^{\nu}-w^{\nu} .
$$

Proof. Let $d^{\nu}=\widehat{S}_{\nu}^{-1}\left(B u^{\nu}-C w^{\nu}-g\right)=-\widehat{S}_{\nu}^{-1} H\left(w^{\nu}\right)$. Utilizing the definitions (2.3) of $H$, the representation (4.9) of $F^{-1}$ and the definitions (4.19) and (4.13) of $\widehat{S}_{\nu}$ and $S\left(c^{\nu}\right)$, respectively, we get

$$
\begin{aligned}
\widehat{S}_{\nu}\left(w^{\nu}+d^{\nu}\right) & =\widehat{S}_{\nu} w^{\nu}-H\left(w^{\nu}\right) \\
& =\widehat{S}_{\nu} w^{\nu}+B T_{c^{\nu}} \widehat{A}_{c^{\nu}}^{-1} T_{c^{\nu}}\left(f-B^{T} w^{\nu}-A c^{\nu}\right)+B c^{\nu}-C w^{\nu}-g \\
& =\left(B T_{c^{\nu}}\right) \widehat{A}_{c^{\nu}}^{-1}\left(T_{c^{\nu}} f-T_{c^{\nu}} A c^{\nu}\right)-\left(g-B c^{\nu}-I\left(c^{\nu}\right) w^{\nu}\right) .
\end{aligned}
$$

Hence, $\tilde{w}^{\nu}=w^{\nu}+d^{\nu}$ is the second component of the solution of (5.4). This completes the proof.

Usually, the active coefficients $c^{\nu}$ of $u^{\nu}$ can be computed much faster than $u^{\nu}$ itself: For nondegenerate problems monotone multigrid methods [31] or even simple projected Gauß-Seidel relaxations [22, Chapter V] provide $c^{\nu}$ in a finite number of steps. Using the a priori estimate (cf., e.g., [29, p. 24])

$$
\left\|u^{*}-u^{\nu}\right\|_{A} \leq\left\|B\left(w^{*}-w^{\nu}\right)\right\|_{A^{-1}}
$$

the accuracy of $u^{\nu}$ can be estimated without actual computation of $u^{\nu}$. 
In order to determine efficient step sizes $\rho^{\nu}$ by Armijo's strategy (cf. Proposition 3.1), we have to evaluate $F^{-1}$ for each test $j=0, \ldots$ in (3.12). Though it is possible to develop straightforward inexact variants of existing damping strategies, e.g., of the Curry-Altmann principle [39, p. 483], an even cheaper heuristic strategy will be applied in the numerical computations to be reported below: We set $\rho^{\nu}=1$, if the condition

$$
\left\|d^{\nu}\right\|_{M} \leq \sigma\left\|d^{\nu-1}\right\|_{M}
$$

holds with some fixed parameter $\sigma \in(0,1)$ and compute $\rho^{\nu}$ according to Armijo's strategy otherwise. Note that it is not hard to show convergence, if (5.6) holds for $d^{\nu}=\widehat{S}_{\nu}^{-1} H\left(w^{\nu}\right)$ and all $\nu \in \mathbb{N}$.

\section{Numerical Results}

In the following examples $\Omega=(0,1) \times(0,1)$ denotes the unit square and the triangulation $\mathcal{T}_{J}$ of $\Omega$ is resulting from $J$ uniform refinement steps as applied to the initial partition $\mathcal{T}_{0}$ consisting of four congruent subtriangles. The uniform refinement $\mathcal{T}_{j+1}$ of $\mathcal{T}_{j}$ is obtained by connecting the midpoints of all triangles $T \in \mathcal{T}_{j}$. Hence, the meshsize of $\mathcal{T}_{J}$ is $h_{J}=2^{-J}$. The sequence $\mathcal{T}_{0} \subset \mathcal{T}_{1} \subset \cdots \subset \mathcal{T}_{J}$ of triangulations gives rise to a nested sequence $\mathcal{S}_{0} \subset \mathcal{S}_{1} \subset \cdots \subset \mathcal{S}_{J}$ of finite element spaces

$$
\mathcal{S}_{j}=\left\{v \in C(\bar{\Omega})|v|_{T} \text { is linear } \forall T \in \mathcal{T}_{j}\right\} \subset H^{1}(\Omega), \quad j=0, \ldots, J .
$$

The standard nodal basis of $\mathcal{S}_{J}$ is denoted by $\lambda_{p}, p \in \mathcal{N}_{J}$, where $\mathcal{N}_{J}$ stands for the set of vertices of $\mathcal{T}_{J}$. Homogeneous Dirichlet conditions give rise to the subspace

$$
\mathcal{S}_{J, 0}=\operatorname{span}\left\{\lambda_{p} \mid p \in \mathcal{N}_{J, 0}\right\} \subset H_{0}^{1}(\Omega), \quad \mathcal{N}_{J, 0}=\mathcal{N}_{J} \cap \Omega .
$$

The scalar product in $L^{2}(\Omega)$ and its lumped version in $\mathcal{S}_{J}$ is denoted by $(\cdot, \cdot)$ and $\langle\cdot, \cdot\rangle$, respectively. The linear space of piecewise constant functions

$$
\mathcal{P}_{J}=\left\{v \in L^{2}(\Omega)|v|_{T} \text { is constant } \forall T \in \mathcal{T}_{J}\right\} \subset L^{2}(\Omega)
$$

is spanned by the canonical basis $\mu_{T}, T \in \mathcal{T}_{J}$, as defined by $\mu_{T}(x)=1$ for $x \in \operatorname{int} T$ and $\mu_{T}(x)=0$ otherwise.

6.1. An optimal control problem with control constraints. For given $y_{0} \in$ $L^{4}(\Omega)$ and $\varepsilon>0$, we consider the following optimal control problem [47].

Find $y \in H_{0}^{1}(\Omega)$ and $u \in L^{\infty}(\Omega)$ such that

$$
\mathcal{J}(y, u)=\int_{\Omega} \frac{1}{2}\left\|y-y_{0}\right\|_{L^{2}(\Omega)}^{2}+\frac{\varepsilon}{2}\|u\|_{L^{2}(\Omega)}^{2} d x
$$

is minimal over all functions in $H_{0}^{1}(\Omega)$ and $L^{\infty}(\Omega)$, subject to the state equation

$$
(\nabla y, \nabla v)=(u, v) \quad \forall v \in H_{0}^{1}(\Omega)
$$

and the control constraint

$$
u \in \mathcal{K}=\left\{v \in L^{\infty}(\Omega)|| v(x) \mid \leq 1, \text { a.e. in } \Omega\right\} .
$$

Approximating $H_{0}^{1}(\Omega)$ by $\mathcal{S}_{J, 0}$ and $\mathcal{K}$ by

$$
\mathcal{K}_{J}=\left\{\left.v \in \mathcal{P}_{J}|| v\right|_{T} \mid \leq 1 \quad \forall T \in \mathcal{T}_{J}\right\} \subset \mathcal{K},
$$

we obtain a discrete analogue of the continuous problem. For existence and error estimates, we refer to [1]. We restrict our considerations to this discretization only. However, the algorithm behaves similar for other discretizations, e.g., with linear 

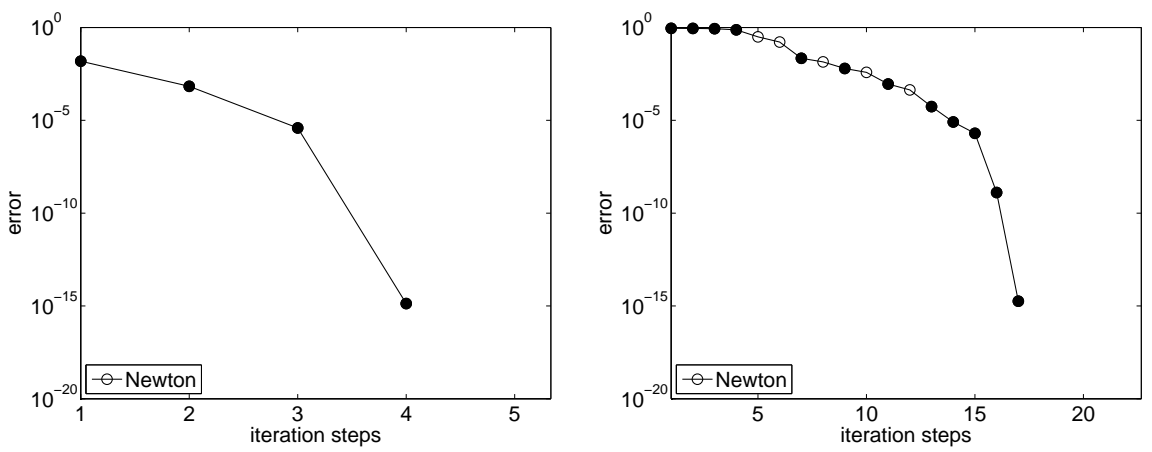

FiguRE 6.1. Iteration history for $\varepsilon=10^{-4}$ (left) and $\varepsilon=10^{-8}$ (right). The filled dots indicate $\rho^{\nu}=1$.

finite elements for the control. After incorporating (6.2) by a Lagrange multiplier $w$, the Kuhn-Tucker conditions of the discretized problem can be rewritten in the form (1.1) with $n=\left|\mathcal{N}_{J, 0}\right|+\left|\mathcal{T}_{J}\right|, m=\left|\mathcal{T}_{J}\right|, F=A+\partial \mathcal{K}_{J}$,

$$
A=\left(\begin{array}{cc}
D_{\mathcal{S}} & 0 \\
0 & \varepsilon D_{\mathcal{P}}
\end{array}\right), \quad D_{\mathcal{S}}=\left(\left\langle\lambda_{p}, \lambda_{q}\right\rangle\right)_{p, q \in \mathcal{N}_{J, 0}}, \quad D_{\mathcal{P}}=\left(\left(\mu_{T}, \mu_{T^{\prime}}\right)\right)_{T, T^{\prime} \in \mathcal{T}_{J}},
$$

$B=\left(\begin{array}{ll}A_{\mathcal{S}} & -D_{\mathcal{S P}}\end{array}\right), \quad A_{\mathcal{S}}=\left(\left(\nabla \lambda_{p}, \nabla \lambda_{q}\right)\right)_{p, q \in \mathcal{N}_{J, 0}}, \quad D_{\mathcal{S P}}=\left(\left(\lambda_{p}, \mu_{T}\right)\right)_{p \in \mathcal{N}_{J, 0}, T \in \mathcal{T}_{J}}$

$C=0$ and suitable right hand sides $f, g$. It is easily checked that the assumptions (A1'), (A2), and (A3) are fulfilled. Moreover, it turns out that $S(c)$ is s.p.d. $\forall c \in \mathcal{C}$. As a consequence, $h$ must be strongly convex (cf. Lemma 4.2) providing uniqueness (A4) and linear convergence of the Newton-type iteration to be called NEwTON as well as its inexact version (cf. Theorem 4.1 and Theorem 4.3). In general, we have $\operatorname{rank} B=m<n$ so that it is not clear from our present analysis that $S_{\nu}=S\left(c^{\nu}\right) \in \partial_{B}\left(H\left(w^{\nu}\right)\right.$ ) (cf. Proposition 4.2). As $A$ is diagonal, the quadratic obstacle problems (5.2) arising in each iteration step can be easily solved by nodal projection. The linear saddle point problems (5.3) are evaluated by the direct solver UMFPACK [15].

Following [43, Chapter 5], we select the desired state

$$
y_{0}(x)=0.001 \begin{cases}4 & \text { if } x \in[0,0.75] \times[0,0.5] \\ -10 & \text { if } x \in[0,0.75] \times[0.5,1] \\ -2 & \text { if } x \in[0.75,1] \times[0,0.5] \\ 50 & \text { if } x \in[0.75,1] \times[0.5,1]\end{cases}
$$

in our numerical computations. The mesh size $h_{J}=2^{-J}$ is resulting from $J=7$ refinement steps. Finally, we choose the parameters

$$
\alpha=10^{-2}, \quad \alpha_{\nu}=\max \left\{1,-\alpha \frac{\left\langle\nabla h\left(w^{\nu}\right), d^{\nu}\right\rangle}{\left\|d^{\nu}\right\|_{M}^{2}}\right\}, \quad \beta=0.5, \quad \delta=0.5
$$

in the associated Armijo strategy (cf. Proposition 3.1).

Figure 6.1 shows the algebraic error $\left\|w^{*}-w^{\nu}\right\|_{M}$ over the number of iteration steps for the two problem parameters $\varepsilon=10^{-4}$ and $\varepsilon=10^{-8}$, respectively. The algebraic error is measured in the energy norm induced by the Schur complement 

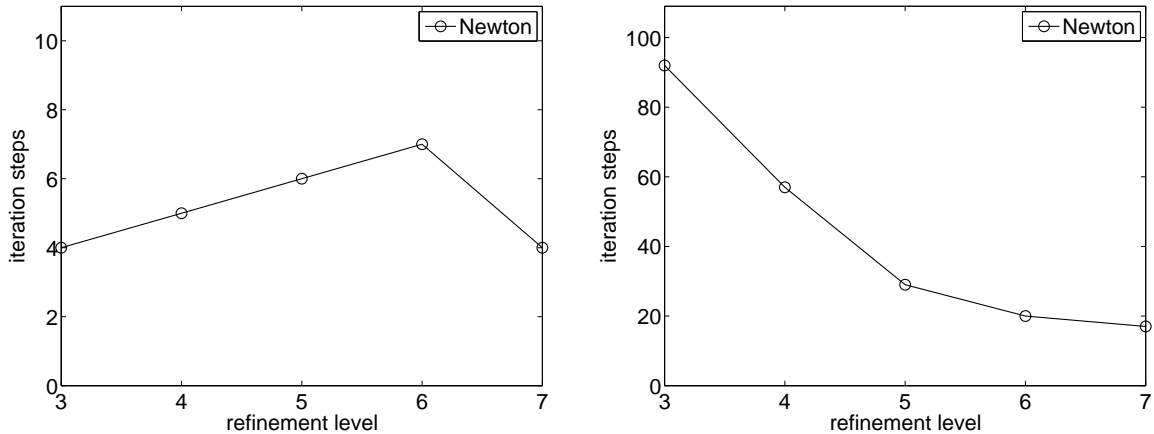

Figure 6.2. Mesh dependence for $\varepsilon=10^{-4}$ (left) and $\varepsilon=10^{-8}$ (right)

$M=B A^{-1} B^{T}$ providing

$$
\left\|w^{*}-w^{\nu}\right\|_{M}=\left\|B^{T}\left(w^{*}-w^{\nu}\right)\right\|_{A^{-1}} \geq\left\|u^{*}-u^{\nu}\right\|_{A}
$$

according to (5.5). The "exact" solution $w^{*}$ is precomputed to roundoff errors. In both cases, we observe superlinear convergence and finite termination, even exceeding the findings of Theorem 4.1. The condition number of (6.1) is increasing for decreasing regularization parameter $\varepsilon$. This is reflected by the large number of iteration steps for the small value $\varepsilon=10^{-8}$. As the solution of the (diagonal!) obstacle problems (5.2) is almost for free and, in addition, no more than two tests are necessary in Armijo damping, almost $100 \%$ of cpu time is consumed by the solution of the linear saddle point problems. For the given initial iterates the undamped algorithm only converges for $\varepsilon=10^{-4}$ but not for $\varepsilon=10^{-8}$ as indicated by Figure 6.1. On the other hand, in both cases the damping parameter $\rho^{\nu}=1$ is accepted before the correct active set is detected in the last iteration step.

We now investigate the mesh dependence of NEWTON. The two pictures in Figure 6.2 show the number of iteration steps required for the solution to roundoff errors over the refinement levels. For both values $\varepsilon=10^{-4}$ and $\varepsilon=10^{-8}$, the convergence speed seems to saturate with increasing refinement. It is interesting that coarser problems seem to become even harder for small $\varepsilon$. Note that the maximal number of Armijo tests is also increasing from two to ten on the coarsest mesh.

6.2. A Cahn-Hilliard problem. For given $\varepsilon>0$, final time $T>0$ and initial condition $u_{0} \in \mathcal{K}=\left\{v \in H^{1}(\Omega)|| v \mid \leq 1\right\}$, we consider the following initial value problem for the Cahn-Hilliard equation with an obstacle potential $[7,11,18]$.

Find $u \in H^{1}\left(0, T ;\left(H^{1}(\Omega)\right)^{\prime}\right) \cap L^{\infty}\left(0, T ; H^{1}(\Omega)\right)$ and $w \in L^{2}\left(0, T ; H^{1}(\Omega)\right)$ with $u(0)=u_{0}$ such that $u(t) \in \mathcal{K}$ and

$$
\begin{array}{cl}
\left\langle\frac{d u}{d t}, v\right\rangle_{H^{1}(\Omega)}+(\nabla w, \nabla v)=0, & \forall v \in H^{1}(\Omega), \\
\varepsilon(\nabla u, \nabla v-\nabla u)-(u, v-u) \geq(w, v-u), & \forall v \in \mathcal{K},
\end{array}
$$

holds for a.e. $t \in(0, T)$.

Here $\langle\cdot, \cdot\rangle_{H^{1}(\Omega)}$ denotes the duality pairing of $H^{1}(\Omega)$ and $H^{1}(\Omega)^{\prime}$. The unknown functions $u$ and $w$ are called order parameter and chemical potential, respectively. 
For existence and uniqueness results we refer to [7]. Semi-implicit Euler discretization in time and finite elements in space $[6,8]$ lead to the following discretized problem.

Find $u_{J}^{k} \in \mathcal{K}_{J}$ and $w_{J}^{k} \in \mathcal{S}_{J}$ such that

$$
\begin{array}{ccrl}
\left\langle u_{J}^{k}, v\right\rangle+\tau\left(\nabla w_{J}^{k}, \nabla v\right) & =\left\langle u_{J}^{k-1}, v\right\rangle, & & \forall v \in \mathcal{S}_{J}, \\
\varepsilon\left(\nabla u_{J}^{k}, \nabla\left(v-u_{J}^{k}\right)\right)-\left\langle w_{J}^{k}, v-u_{J}^{k}\right\rangle \geq\left\langle u_{J}^{k-1}, v-u_{J}^{k}\right\rangle, & & \forall v \in \mathcal{K}_{J} .
\end{array}
$$

holds for each $k=1, \ldots, N$.

We have chosen a uniform time step size $\tau=T / N$, and $\mathcal{K}_{J}=\mathcal{K} \cap \mathcal{S}_{J}$ is the nodal approximation of $\mathcal{K}$. The initial condition $u_{J}^{0} \in \mathcal{K}_{J}$ is obtained by discrete $L^{2}$ projection $\left\langle u_{J}^{0}, v\right\rangle=\left(u_{0}, v\right) \forall v \in \mathcal{S}_{J}$. Existence, uniqueness and error estimates have been established in [8]. More precisely, there exists a discrete solution $\left(u_{J}^{k}, w_{J}^{k}\right)$ with uniquely determined $u_{J}^{k}, k=1, \ldots, N$. Moreover, $w_{J}^{k}$ is also unique, provided that the condition

$$
\exists p \in \mathcal{N}_{J}: \quad\left|u_{J}^{k}(p)\right|<1
$$

is fulfilled. Hence, (A4) is satisfied in this case. If (6.7) is violated, then either the triangulation $\mathcal{T}_{J}$ is too coarse to resolve the diffuse interface or only one phase is present, i.e., $u_{J}$ is constant. For the iterative solution of each spatial problem (6.6) a projected block Gauß-Seidel scheme [6] and an ADI-type iteration [35] are widely used. Both algorithms suffer from rapidly deteriorating convergence rates for increasing refinement.

Exploiting discrete mass conservation $\left\langle u_{J}^{k}, 1\right\rangle=\left(u_{0}, 1\right)$, each spatial problem (6.6) takes the form (1.1) with $n=m=\left|\mathcal{N}_{J}\right|, F=A+\partial I_{\mathcal{K}_{J}}$,

$$
\begin{gathered}
A=\varepsilon\left(\left\langle\lambda_{p}, 1\right\rangle\left\langle\lambda_{q}, 1\right\rangle+\left(\nabla \lambda_{p}, \nabla \lambda_{q}\right)\right)_{p, q \in \mathcal{N}_{J}}, \\
B=-\left(\left\langle\lambda_{p}, \lambda_{q}\right\rangle\right)_{p, q \in \mathcal{N}_{J}}, \quad C=\tau\left(\left(\nabla \lambda_{p}, \nabla \lambda_{q}\right)\right)_{p, q \in \mathcal{N}_{J}} .
\end{gathered}
$$

and suitable right hand sides $f$ and $g$. Assuming (6.7), it is easily checked that the assumptions (A1'), (A2), and (A3) are satisfied. Observe that $A$ is the sum of a sparse stiffness matrix and a rank one matrix. We clearly have $\operatorname{rank} B=n$ so that $S(c) \in \partial_{B} H(w)$ is a B-subdifferential of $H$ (cf. Proposition 4.2). However, as $C$ is only positive semidefinite, the kernel ker $S(c)$ is trivial only if $N_{c}^{\bullet} \neq N$. In the singular case $N_{c}^{\bullet}=N$, ker $S(c)$ is spanned by the constant vector $k_{1}=(1, \ldots, 1)^{T}$.

For our numerical computations, we select $\varepsilon=10^{-4}$, the time step $\tau=\varepsilon$, and the mesh size $h_{J}=2^{-J}$ is resulting from $J=9$ refinement steps. The initial condition $u_{0}$ takes the values $u_{0}(x)=\max \left\{\min \left\{2 \sin \left(4 \pi x_{1}\right) \sin \left(4 \pi x_{2}\right), 1\right\},-1\right\}$.

We compare the nonsmooth Newton-like method (cf. Theorem 4.2) called NEwTON-LIKE, the inexact variant (cf. Theorem 4.3) called INEXACT and projected block Gauß-Seidel relaxation [6] called GAUSS-SEIDEL. The actual active coefficients are computed from the obstacle problem (5.2) by a monotone multigrid method [31]. The linear saddle point problems (5.4) are solved iteratively by a linear multigrid method with block Gauß-Seidel smoother and canonical restriction and prolongation. In the exact version NEWTON-LIKE the solution $w^{\nu}$ is computed to machine accuracy, and we use Armijo damping (cf. Proposition 3.1) with $\delta=10^{-3}$ and the other parameters given in (6.4). In the $\nu$-th outer iteration of INEXACT we apply $3 \nu$ steps of the linear multigrid method with $V(3,3)$ cycle to match the asymptotic accuracy condition (3.17), and we use heuristic damping (5.6) with $\sigma=0.5$. 

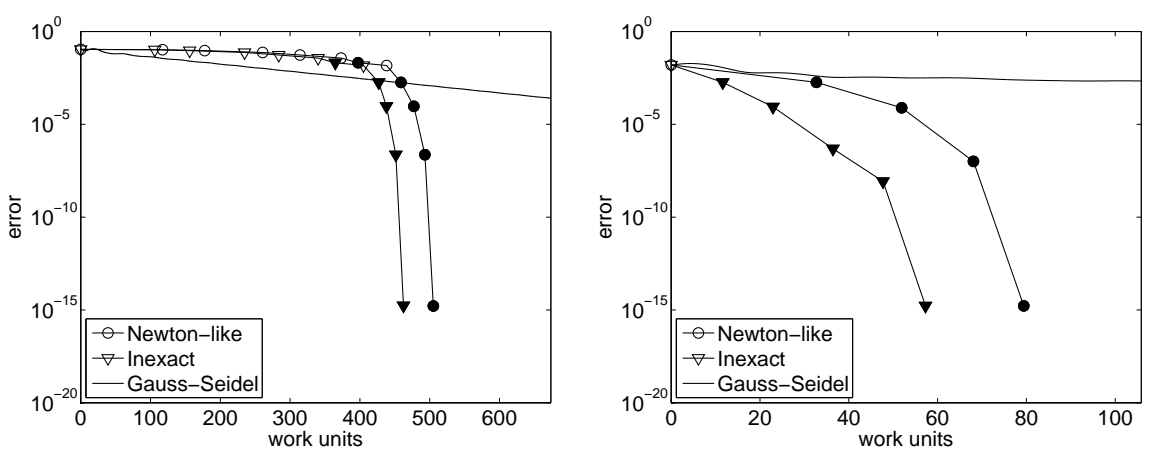

Figure 6.3. Iteration histories for good initial iterates (left) and bad initial iterates (right). The filled dots indicate $\rho^{\nu}=1$.

Figure 6.3 illustrates the algebraic error $\left\|w^{*}-w^{\nu}\right\|_{M}$ over the computational work for the first two spatial problems. We choose the discrete $H^{1}$-norm induced by $M=D+C$ with $D=\tau\left(\left\langle\lambda_{p}, \lambda_{q}\right\rangle\right)_{p, q \in \mathcal{N}_{J}}$. Hence, $\left\|u^{*}-u^{\nu}\right\|_{A} \leq c\left\|w^{*}-w^{\nu}\right\|_{M}$ with a constant $c$ independent of $J$ (cf. (5.5) and Poincaré's inequality). The "exact" solution $w^{*}$ is precomputed to roundoff errors. For a fair comparison, the computational work is now measured in work units (not in iteration steps). One work unit is the cpu time required by one linear multigrid $V(3,3)$ cycle as applied to the linear saddle point problem (5.4). The left and the right picture in Figure 6.3 show the iteration histories for the spatial problems arising from the first and the second time step, respectively. Each marker refers to one iteration step of NEWTONLIKE and INEXACT, respectively. As no initial data are available for the chemical potential $w$, we start with the bad initial iterate $w^{0}=0$ in the first problem while the final approximation from the previous time step provides a reasonable initial for the second one. This makes quite a difference. For the bad initial iterate, it takes about 400 work units (about 6 iteration steps) until NEWTON-LIKE and INEXACT finally display superlinear convergence. GAUSS-SEIDEL is even more efficient in the beginning of the iteration, but not comparable later. For reasonable initial iterates, superlinear convergence starts immediately (observe the different scaling of the $x$ axis). In both cases, INEXACT turns out to be more efficient than NEWTON-LIKE.

Table 1 gives more detailed insight into the performance of the different building blocks of INEXACT as applied to the first problem. The number of tests involved

\begin{tabular}{|l|l|l|l|l|l|l|l|l|l|l|l|}
\hline INEXACT & 1 & 2 & 3 & 4 & 5 & 6 & 7 & 8 & 9 & 10 & 11 \\
\hline \hline \# tests & 7 & 3 & 5 & 3 & 3 & 1 & 3 & 1 & 0 & 0 & 0 \\
\hline \hline \% Armijo & 88.7 & 85.9 & 88.1 & 76.1 & 74.2 & 49.2 & 69.3 & 44.4 & 0.1 & 0.1 & 0.1 \\
\hline \% obstacle & 7.2 & 0.0 & -0.0 & -0.0 & 0.0 & 0.0 & 0.0 & -0.0 & 0.0 & 27.2 & 24.0 \\
\hline \% linear & 4.1 & 14.0 & 11.9 & 23.8 & 25.7 & 50.7 & 30.7 & 55.5 & 99.7 & 72.6 & 75.7 \\
\hline \hline work units & 106.1 & 50.1 & 78.5 & 49.0 & 56.4 & 24.5 & 40.5 & 21.8 & 11.0 & 13.4 & 10.9 \\
\hline
\end{tabular}

TABle 1. Distribution of cpu time over the subtasks in each Uzawa step 

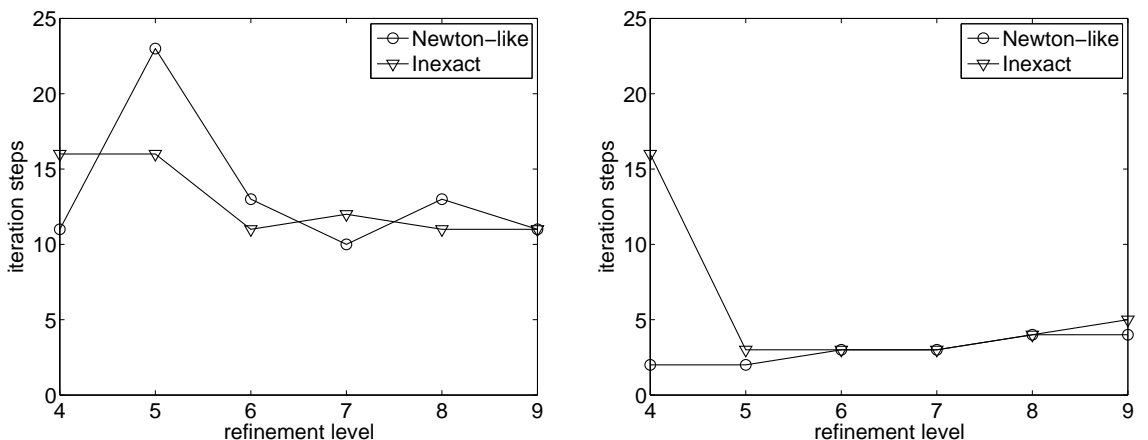

Figure 6.4. Mesh dependence for good initial iterates (left) and bad initial iterates (right)

in Armijo damping is given in the first line. Due to the bad initial iterate, a considerable number of tests is required in the beginning which later goes down to zero. The following three lines show the actual percentage of cpu time required by damping and the approximate solution of the obstacle problem and of the linear saddle point problem, respectively. These numbers do not sum to 100 , because minor computations are neglected. Observe that the computational work is first dominated by Armijo damping and later by the increasing number of multigrid sweeps for the linear saddle point problem. Apart from the initial step, the detection of the active set takes not more than 5 monotone multigrid sweeps, each of which is cheaper than a multigrid sweep for the linear saddle point problem. As shown in the last line, the absolute amount of computational work strongly depends on the number of Armijo tests which in turn strongly depends on the (problem dependent!) choice of the parameters. Hence, the performance of INEXACT could be probably improved by more careful tuning the damping parameters. Observe that for bad initial iterates neither the exact nor the inexact method converges without damping. On the other hand, for both versions the damping parameter $\rho^{\nu}=1$ is accepted before the correct active set is detected (cf. Figure 6.3). More efficient affineinvariant damping strategies for nonsmooth Newton-type algorithms will be the subject of future research.

We now investigate the mesh dependence of Newton-Like and IneXact. Figure 6.4 shows the number of iteration steps required for the solution to roundoff errors over the refinement levels. For the first spatial problem (left), we always start with $w^{\nu}=0$, while for the second spatial problem, (right), we always start from the previous time level. In both cases, the overall convergence speed seems to be scarcely affected by decreasing mesh size. It is astonishing that INEXACT sometimes even needs less iteration steps. Note that the averaged error reduction per work unit of INEXACT is about $\rho=0.6$. We observed $\rho \approx 0.16$ for the linear multigrid solver as applied to the linear saddle point problems. Hence, for reasonable initial iterates, the solution of the discrete Cahn-Hilliard problem by straightforward inexact versions required about three to four times the cpu time for the solution of related linear saddle point problems by standard multigrid methods. 
GRÄSER AND KORNHUBER

\section{REFERENCES}

[1] N. Arada, E. Casas, and F. Tröltzsch. Error estimates for the numerical approximation of a semilinear elliptic control problem. Comput. Optim. Appl., 23(2):201-229, 2002.

[2] L. Armijo. Minimization of functions having Lipschitz-continuous first partial derivatives. Pazific J. Math., 204:126-136, 1966.

[3] L. Badea. Convergence rate of a Schwarz multilevel method for the constrained minimization of nonquadratic functionals. SIAM J. Numer. Anal., 44(2):449477, 2006.

[4] L. Badea, X.-Ch. Tai, and J. Wang. Convergence rate analysis of a multiplicative Schwarz method for variational inequalities. SIAM J. Numer. Anal., 41(3):1052-1073, 2003.

[5] J.W. Barrett and J.F. Blowey. An error bound for the finite element approximation of the cahn-hilliard equation with logarithmic free energy. Numerische Mathematik, 72:1-20, 1995.

[6] J.W. Barrett, R. Nürnberg, and V. Styles. Finite element approximation of a phase field model for void electromigration. SIAM J. Numer. Anal., 42(2):738$772,2004$.

[7] J.F. Blowey and C.M. Elliott. The Cahn-Hilliard gradient theory for phase separation with non-smooth free energy Part I: Mathematical analysis. European J. Appl. Math., 2:233-280, 1991.

[8] J.F. Blowey and C.M. Elliott. The Cahn-Hilliard gradient theory for phase separation with non-smooth free energy Part II: Numerical analysis. European J. Appl. Math., 3:147-179, 1992.

[9] D. Braess and R. Sarazin. An efficient smoother for the Stokes problem. Appl. Numer. Math., 23(1):3-19, 1997.

[10] M. Brokate and J. Sprekels. Hysteresis and Phase Transition. Number 121 in Applied mathematical sciences. Springer, Berlin Heidelberg New York, 1996.

[11] J.W. Cahn and J.E. Hilliard. Free energy of a nonuniform system I. Interfacial energy. J. Chem. Phys., 28:258-267, 1958.

[12] X. Chen. On preconditioned Uzawa methods and SOR methods for saddlepoint problems. J. Comput. Appl. Math., 100(2):207-224, 1998.

[13] F.H. Clarke. Optimization and Nonsmooth Analysis. John Wiley and Sons, New York, 1983.

[14] R.W. Cottle, J.S. Pang, and R.E. Stone. The Linear Complentary Problem. Academic Press, Boston, 1992.

[15] T. A. Davis. Algorithm 832: Umfpack v4.3 - an unsymmetric-pattern multifrontal method. ACM Trans. Math. Softw., 30(2):196-199, 2004.

[16] P. Deuflhard. Newton Methods for Nonlinear Problems. Springer, Berlin, Heidelberg, 2004.

[17] I. Ekeland and R. Temam. Convex Analysis. North-Holland, Amsterdam, 1976.

[18] C.M. Elliott. The Cahn-Hilliard model for the kinetics of phase separation. In J.F. Rodrigues, editor, Mathematical models for phase change problems, pages 35 - 73, Basel, 1989. Birkhäuser.

[19] H. Garcke and B. Stinner. Second order phase field asymptotics for multicomponent systems. Interf. Free Bound., 8:131-157, 2006. 
[20] D. Gilbarg and N.S. Trudinger. Elliptic Partial Differential Equations of Second Order. Springer, Berlin, 2. edition, 1988.

[21] R. Glowinski. Numerical Methods for Nonlinear Variational Problems. Springer Series in Computational Physics. Springer, Berlin Heidelberg New York, 3. edition, 1984.

[22] R. Glowinski. Numerical Methods for Nonlinear Variational Problems. Springer, New York, 1984.

[23] C. Gräser. Globalization of nonsmooth newton methods for optimal control problems. 2007. submitted.

[24] C. Gräser and R. Kornhuber. On preconditioned uzawa-type iterations for a saddle point problem with inequality constraints. Preprint A-5-05, FU Berlin, 2005. to appear in Proc. XVI Int. Conf. on Domain Decomposition.

[25] C. Gräser and R. Kornhuber. Adaptive multigrid methods for the CahnHilliard equation with logarithmic potential. 2008. in preparation.

[26] C. Gräser, R. Kornhuber, and X.-Ch. Tai. Multigrid methods for obstacle problems. 2008. in preparation.

[27] M. Hintermüller, K. Ito, and K. Kunisch. The primal-dual active set strategy as a semismooth Newton method. SIAM J. Optim., 13(3):865-888, 2003.

[28] Q. Hu and J. Zou. Nonlinear inexact Uzawa algorithms for linear and nonlinear saddle-point problems. SIAM J. Optim., 16(3):798-825, 2006.

[29] D. Kinderlehrer and G. Stampacchia. An Introduction to Variational Inequalities and Their Applications. Academic Press, New York, 1980.

[30] R. Kornhuber. Monotone multigrid methods for elliptic variational inequalities I. Numer. Math., 69(2):167-184, 1994.

[31] R. Kornhuber. Monotone multigrid methods for elliptic variational inequalities I. Numer. Math., 69:167 - 184, 1994.

[32] R. Kornhuber. On constrained Newton linearization and multigrid for variational inequalities. Numer. Math., 91:699-721, 2002.

[33] R. Kornhuber and R. Krause. Robust multigrid methods for vector-valued allen-cahn equations with logarithmic free energy. Comput. Vis. Sci., 9:103$116,2006$.

[34] J.L. Lions. Optimal control of systems governed by partial differential equations. Springer, Berlin Heidelberg New York, 1971.

[35] P.L. Lions and B. Mercier. Splitting algorithms for the sum of two nonlinear operators. Siam J. Numer. Anal., 16:964-979, 1979.

[36] J. Mandel. A multilevel iterative method for symmetric, positive definite linear complementarity problems. Appl. Math. Optimization, 11:77-95, 1984.

[37] A. Nekvinda and L. Zajíček. A simple proof of the Rademacher theorem. Časopis Pěst. Mat, 113(4):337-341, 1988.

[38] J. Nocedal. Theory of algorithms for unconstrained optimization. Acta Numerica, 1:199-242, 1992.

[39] J.M. Ortega and W.C. Rheinboldt. Iterative Solution of Nonlinear Equations in Several Variables. Academic Press, New York, 1970.

[40] M.J.D. Powell. Direct search algorithms for optimization calculations. Acta Numerica, 7:287-336, 1998.

[41] L. Qi. Convergence analysis of some algorithms for solving nonsmooth equations. Mathematics of Operations Research, 18(1):227-244, 1993. 
[42] L. Qi and J. Sun. A nonsmooth version of Newtons's method. Mathematical Programming, 58:353-367, 1993.

[43] A. Schiela and M. Weiser. Superlinear convergence of the control reduced interior point method for pde contrained optimization. Technical report, Zuse Institute Berlin, 2005. to appear in COAP.

[44] J. Schöberl and W. Zulehner. On Schwarz-type smoothers for saddle point problems. Numer. Math., 95(2):377-399, 2003.

[45] J.C. Simo and T.J.R. Hughes. Computational inelasticity. Springer, Berlin, 1998.

[46] X.-Ch. Tai. Rate of convergence for some constraint decomposition methods for nonlinear variational inequalities. Numer. Math., 93(4):755-786, 2003.

[47] Fredi Tröltzsch. Optimale Steuerung partieller Differentialgleichungen. Theorie, Verfahren und Anwendungen. Vieweg, Wiesbaden, 2005.

[48] M. Ulbrich. Nonsmooth newon-like methods for variational inequalities and constrained optimization problems in function spaces. Habilitationsschrift, TU München, 2002.

[49] S.P. Vanka. Block-implicit multigrid solution of Navier-Stokes equations in primitive variables. J. Comput. Phys., 65:138-158, 1986.

[50] A. Visintin. Models of Phase Transitions. Birkhäuser, Boston, 1996.

[51] C. Wieners. Nonlinear solution methods for infinitesimal perfect plasticity. ZAMM, 87:643-660, 2007.

[52] S.J. Wright. Primal-dual interior point-methods. SIAM, Philadelphia, 1997.

[53] Y. Ye. Interior point algorithms. Wiley, Chichester, 1997.

[54] W. Zulehner. A class of smoothers for saddle point problems. Computing, 65(3):227-246, 2000.

[55] W. Zulehner. Analysis of iterative methods for saddle point problems: A unified approach. Math. Comput., 71(238):479-505, 2002.

Carsten Gräser, Freie Universität Berlin, Institut für Mathematik II, Arnimallee 2 6, D - 14195 Berlin, Germany

E-mail address: graeser@math.fu-berlin.de

Prof. Dr. Ralf Kornhuber, Freie Universität Berlin, Institut für Mathematik II, Arnimallee 2-6, D - 14195 Berlin, Germany

E-mail address: kornhuber@math.fu-berlin.de 